\title{
A laser ablation source for offline ion production at LEBIT
}

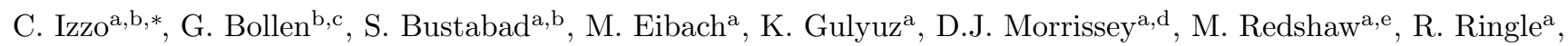 \\ R. Sandler ${ }^{a, b}$, S. Schwarz ${ }^{a}$ A.A. Valverde ${ }^{a, b}$ \\ ${ }^{a}$ National Superconducting Cyclotron Laboratory, East Lansing, Michigan 48824, USA \\ ${ }^{b}$ Department of Physics and Astronomy, Michigan State University, East Lansing, Michigan 48824, USA \\ ${ }^{c}$ Facility for Rare Isotope Beams, East Lansing, Michigan 48824, USA \\ ${ }^{d}$ Department of Chemistry, Michigan State University, East Lansing, Michigan 48824, USA \\ ${ }^{e}$ Department of Physics, Central Michigan University, Mount Pleasant, Michigan 48859, USA
}

\begin{abstract}
A laser ablation ion source has been developed and implemented at the Low-Energy Beam and Ion Trap (LEBIT) facility at the National Superconducting Cyclotron Laboratory. This offline ion source enhances the capabilities of LEBIT by providing increased access to ions used for calibration measurements and checks of systematic effects as well as stable and long-lived ions of scientific interest. The design of the laser ablation ion source and a demonstration of its successful operation are presented.
\end{abstract}

Keywords: laser ablation, ion source, Penning trap, mass measurements, carbon clusters

\section{Introduction}

The Low-Energy Beam and Ion Trap (LEBIT) facility [1] at the National Superconducting Cyclotron Laboratory (NSCL) is the only Penning trap mass spectrometry system currently installed at a projectile-fragmentation facility. This presents a unique opportunity to perform precision mass measurements of rare isotopes that are difficult to produce at other rare-isotope facilities. LEBIT also includes two offline ion sources: a thermal ion source and the recently commissioned laser ablation source (LAS). A schematic of the LEBIT facility is shown in Fig. 1. A 90 degree electrostatic bender allows ions to be transported from the Coupled Cyclotron Facility or either of the two offline sources towards the cooler/buncher, a twostage buffer-gas-filled radiofrequency quadrupole ion trap, which produces cooled ion bunches with low energy and low emittance [2]. The ion bunches are then transported to the 9.4-T Penning trap mass spectrometer, with nonisobaric contaminants suppressed by their time-of-flight using a fast electrostatic deflector along the way [3]. This method can achieve a resolving power of more than 400 . Measurements are performed using the time-of-flight ion cyclotron resonance (TOF-ICR) technique [4] to measure the cyclotron frequency $\nu_{c}$ of the ions, from which the atomic mass of the ions can be precisely determined.

The addition of the LAS provides increased access to ions of stable and long-lived isotopes using a simple offline production method. Similar ion sources have been successfully employed at several other Penning trap facil-

\footnotetext{
* Corresponding author

Email address: izzo@nscl.msu.edu (C. Izzo)
}

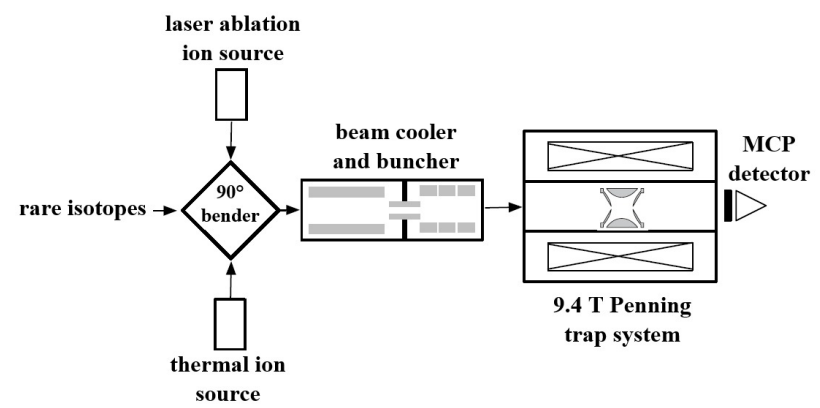

Figure 1: Schematic showing the layout of the LEBIT facility.

ities [5, 6, 7, 8]. The wide array of available stable isotopes with well known masses allows detailed checks of the experimental apparatus and an evaluation of systematic effects [9. In some cases, these effects may even be reduced by the large range of reference ions available with the LAS. Mass-dependent systematic effects scale approximately linearly with the difference in mass-to-charge ratio between the ion of interest and the reference ion, and the uncertainty of the reference ion contributes to the uncertainty of the final mass determination; thus it is important to have access to ions of well known masses spanning a broad mass range. Carbon cluster ions make particularly good reference ions because they contain essentially zero mass uncertainty and span the nuclear chart in intervals of $12 \mathrm{u}$. The LAS provides access to such carbon cluster ions at the LEBIT facility, allowing selection of reference ions that will minimize systematic uncertainties. Finally, the LEBIT LAS has provided access to stable and long-lived isotopes of scientific interest for areas such as neutrino physics [10, 11]. 
The LAS employs a Quantel Brilliant laser to produce pulsed laser light. The Brilliant laser is a pulsed neodymium-doped yttrium aluminum garnet (Nd:YAG) laser with a second harmonic module for outputting 532 $\mathrm{nm}$ light. It achieves a maximum energy of $160 \mathrm{~mJ}$ per pulse, with a pulse duration of $4 \mathrm{~ns}$ and a repetition rate of $20 \mathrm{~Hz}$. The beam spot on the ablation target has a sub-mm diameter, and the laser power is typically limited to well below the maximum in order to achieve a power density of $\sim 5 \times 10^{8} \mathrm{~W} / \mathrm{cm}^{2}$, which is sufficient for ion production. This laser was repurposed from its previous use for characterizing the gas cell facility at the NSCL [12].

Ions are produced by irradiating a solid target with laser light. When a pulse of laser light strikes the target, part of the target material evaporates due to the high fluence of the laser pulse. The laser irradiation induces a high surface temperature, resulting in the emission of positive ions and electrons from the surface, followed by a multistage plasma expansion. A detailed theoretical description of this process is given in Ref. 13 .

During ion production, the laser power is adjusted to maintain a steady count rate. If large shot-to-shot count rate fluctuations are observed, ions can be accumulated in the buncher for several laser shots to achieve a more consistent number of detected counts in each ion bunch. Ion beam intensity can be increased by increasing laser power, but is limited to sub-pA by breakdown of the high voltage when the pressure buildup from ablated material becomes too large. By decreasing the laser power and detuning the steering voltages in the 90 degree electrostatic bender, the average ion count may be reduced to as low as one ion per shot.

\section{Design considerations}

An important design consideration for the LAS is the angle of incidence of the laser light relative to the ablation target. A nonzero angle of incidence can facilitate laser entry and minimize back reflected light to the laser, however the possibility of a nonzero angle of incidence required investigation to determine whether this would significantly decrease the laser power transmitted to the target. Slight losses could be compensated by operating the laser at a higher power, however too much compensation could cause damage to the optical elements. The extent of this effect is given by the Fresnel equations, as shown in Fig. 2. Here the calculated transmittance is plotted as a function of angle of incidence for $\mathrm{S}$ and $\mathrm{P}$ polarized light. Titanium, carbon, and molybdenum targets are shown as examples, with complex refractive indices found in 14, 15, 16, respectively. A Sigradur G target was used for carbon production, and the refractive index of glassy carbon was used to approximate that of Sigradur G. For S polarized light, transmittance falls off with increasing angle of incidence, while for $\mathrm{P}$ polarized light, transmittance does not fall below that at zero degrees except at very large angles. Based
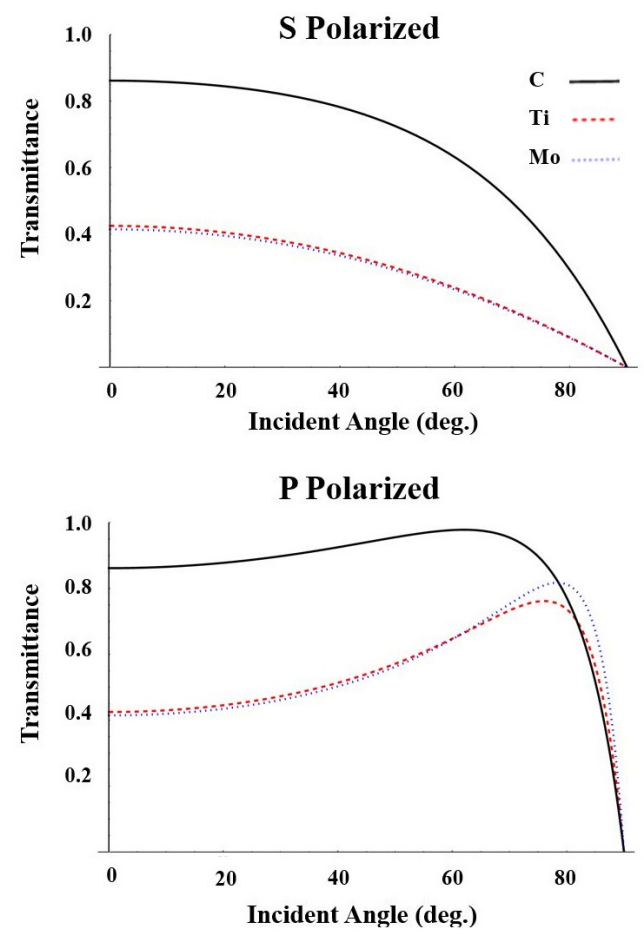

Figure 2: Transmittance plotted as a function of incident angle. Calculations were performed using Fresnel equations with refractive indices for titanium, carbon, and molybdenum targets using $532 \mathrm{~nm}$ light with S polarization (top) and P polarization (bottom).

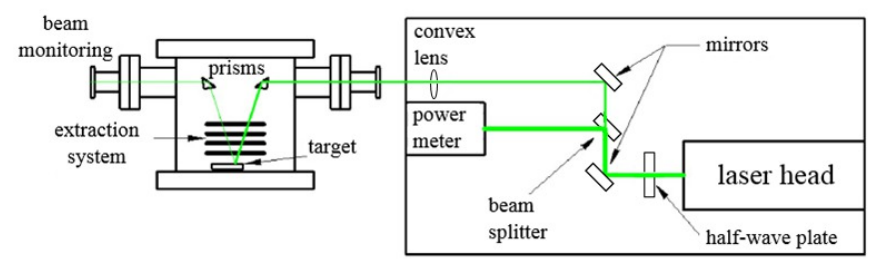

Figure 3: Schematic showing the source design and optical elements used for ablation.

on these results, it was decided to use $\mathrm{P}$ polarized light with an 18 degree angle of incidence for the LAS.

A schematic of the layout of the LAS is presented in Fig. 3. A half-wave plate was placed immediately following the Brilliant laser to convert the laser light from $\mathrm{S}$ to $\mathrm{P}$ polarization. Two mirrors were used to reflect the laser light 90 degrees twice; this helps with confining the laser optics in a relatively small space and with alignment to place the laser beam spot at the desired position on the target. A beam splitter was located between the two mirrors to reflect $80 \%$ of the laser energy to a power meter. This is useful for both monitoring and reducing the laser power, as the maximum energy of $160 \mathrm{~mJ} /$ pulse is substantially more than the pulse energy required for ion production. A single convex lens with a focal length of $335 \mathrm{~mm}$ (for $532 \mathrm{~nm}$ light) was used for laser focusing. The light enters the vacuum chamber parallel to the target through an N-BK7 glass optical window with a $532 \mathrm{~nm}$ antireflective coating. Two right angle prisms were housed 

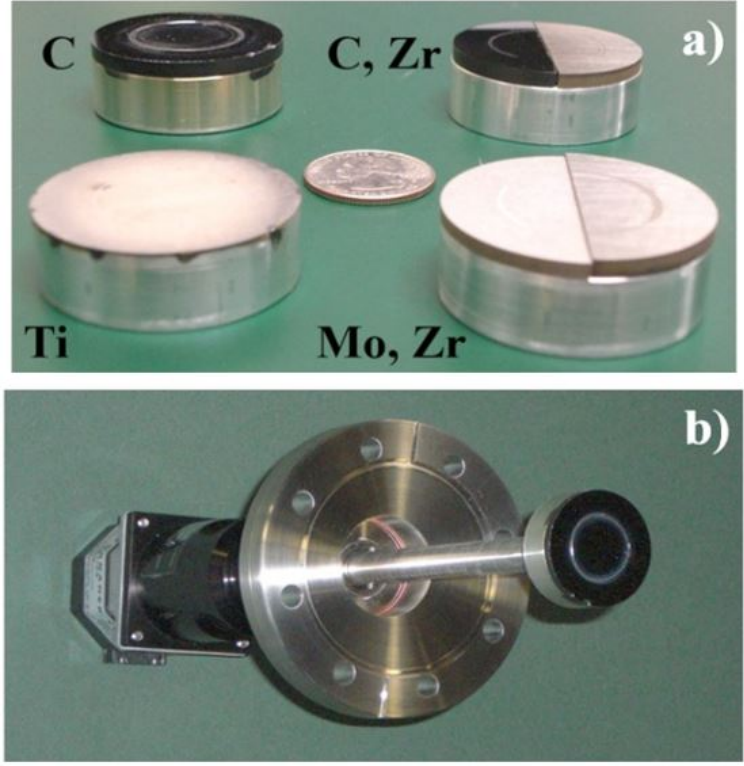

Figure 4: a) Targets used for laser ablation. On the left are single element targets of carbon and titanium, and on the right are two element targets of carbon/zirconium and molybdenum/zirconium. A U.S. quarter coin has been included in the picture for size reference. b) Carbon target mounted on the rotation system. The $20 \mathrm{~mm}$ diameter circular track eroded by the laser when the target is rotated is visible.

inside the vacuum chamber; the first reflects light to the target with an 18 degree angle of incidence, the second was mounted on the opposite side of the chamber and is used to view the laser beam spot on the target.

The process of laser ablation creates craters on the surface of the target, which will result in significant variation in ion yield if the location of the beam spot on the target remains constant. One option is to continuously move the laser beam so that it strikes the target at many different locations. However, this results in ion production at many locations relative to the ion optics, significantly complicating the process of ion extraction. Instead, the geometric center of the circular target was offset from the axis of the laser by $10 \mathrm{~mm}$, and the target was mounted on a rotating shaft. Thus the target was rotated with respect to the beam spot, typically at about $20 \mathrm{rpm}$, while the point of ion production remained fixed.

Fig. 4 a shows four different laser ablation targets used with the LAS at LEBIT. As can be seen in this figure, it is sometimes useful to mount two target materials simultaneously to facilitate the transition between ion production of different elements. Target materials are glued to an aluminum cylinder mount using EPO-TEK 353ND-T. The aluminum cylinder has a threaded hole in the center, which connects to a stainless steel shaft. This allows for fast and easy target switching by simply swapping aluminum mounts. A Powermax stepper motor and a Kollmorgen driver were used to control the rotation such that only the desired element was ablated at a given time. The switching process has been incorporated into the LEBIT
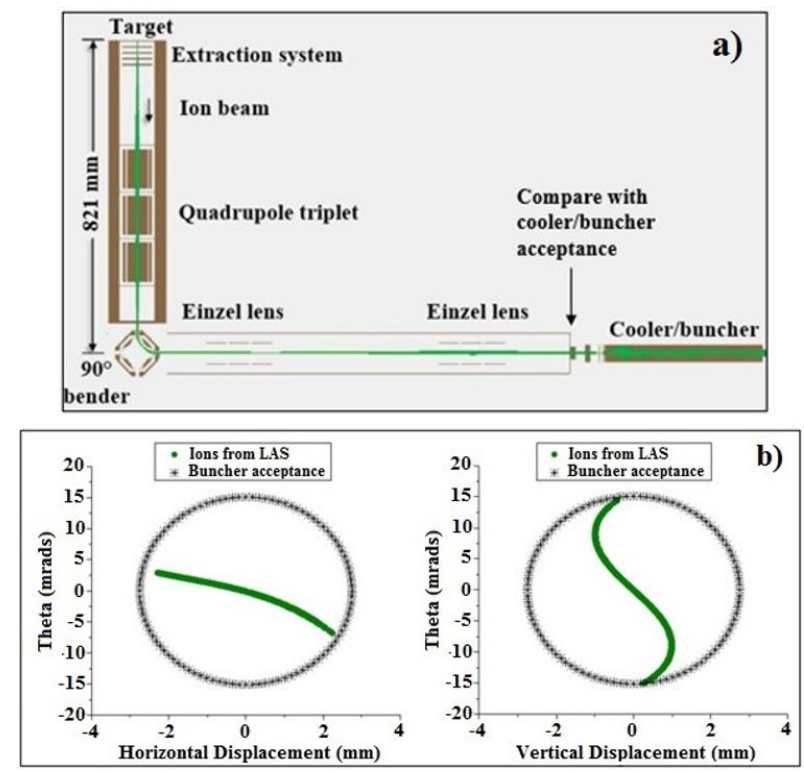

Figure 5: a) Simulation model used to investigate the ion optics necessary for extraction and focusing of a monoenergetic $5 \mathrm{keV}$ beam. b) Simulation results comparing beam properties (green circles) directly before the cooler/buncher with properties required for buncher acceptance (black asterisks). Results indicate that ions produced with the LAS are fully accepted into the cooler/buncher.

PLC system. Fig. 4b shows a carbon target mounted to the rotation system, and the track eroded by the laser when the target is rotating is visible.

A four ring electrostatic extraction system is employed directly in front of the target in order to accelerate the ions to $5 \mathrm{keV}$. This is followed by an electrostatic quadrupole triplet, which provided focusing before the 90 degree bender. Two additional Einzel lenses were then used for focusing ions before entering the cooler/buncher. SIMION software [17] was used to simulate the ion optics for transport from the ablation target to the cooler/buncher; the simulation model used is shown in Fig. 5a. Initial conditions for laser ablated ions are not well understood, but the velocity distribution is expected to be conoidal in direction and to follow the envelope of a Maxwell distribution and the phase explosion phase [18]. For this simulation, ions originated from a single point at the center of the vacuum chamber with a typical initial energy of $3 \mathrm{eV}$ and initial velocities varying in direction over a 45 degree hollow cone. After optimization of the simulated ion optics, beam properties immediately before the cooler/buncher were compared to those required for acceptance into the cooler/buncher. The results of this comparison are shown in Fig 5b and indicate full acceptance of ions produced with the LAS in this model.

The target was mounted with a high voltage break in order to provide the $5 \mathrm{kV}$ difference necessary to accelerate the ions to $5 \mathrm{keV}$. This allows the target rotation system to remain at true ground potential during normal offline operation, while the beamline prior to the cooler/buncher operates on the normal $-5 \mathrm{kV}$ bias. 


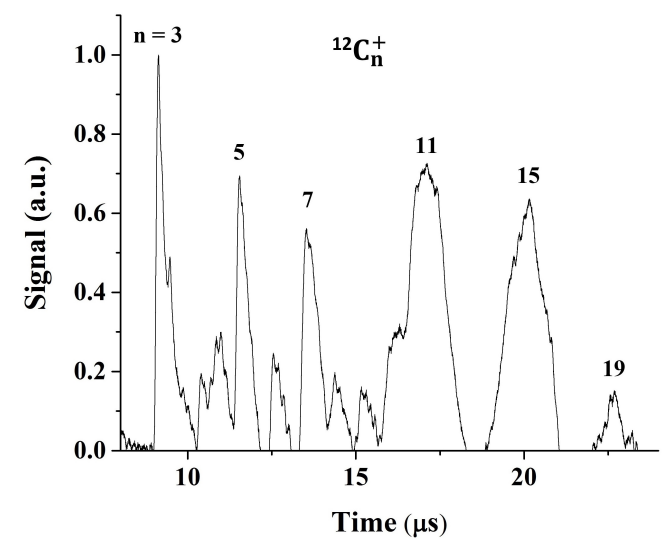

Figure 6: Spectrum of singly charged ${ }^{12} \mathrm{C}$ cluster ions detected on an MCP detector immediately after the 90 degree bender. Peaks are identified by their relative times of flight.

\section{Production of carbon cluster ions}

Ions of ${ }^{12} \mathrm{C}$ clusters are useful for probing systematic effects in Penning traps, and as absolute mass references, because the atomic mass of ${ }^{12} \mathrm{C}$ has zero uncertainty, and binding energies [19, 20, and ionization potentials [21, 22] of carbon clusters are known well enough to not significantly contribute to the uncertainties in mass comparisons, even at $10^{-10}$ relative precision. The set of carbon cluster ions also spans the entire nuclear chart, allowing the investigation of systematic effects over the required range of mass-to-charge ratios 9]. These qualities also make carbon clusters excellent references during on-line measurements.

A Sigradur G carbon target was ablated, and singly charged ions were detected on a micro channel plate (MCP) detector immediately following the 90 degree bender. The resulting spectrum is shown in Fig. 6, where different ${ }^{12} \mathrm{C}$ clusters were identified by their relative times of flight. Relative abundances of the different clusters are sensitive to the laser power, with higher power tending to produce smaller clusters. Preferential selection of certain clusters, such as the abundance of odd clusters over even clusters, can be understood from relative binding energies [19, 20]

When a particular cluster is needed, the laser power can be adjusted to increase the relative production of the desired cluster. RF amplitudes in the cooler/buncher can also be tuned to optimize transport of the desired mass. After cooling and bunching, a certain cluster is selected by its time of flight using a fast electrostatic deflector 3] and sent to the Penning trap for measurement. So far, the clusters ${ }^{12} \mathrm{C}_{3}-{ }^{-12} \mathrm{C}_{9}$ and ${ }^{12} \mathrm{C}_{12}-{ }^{12} \mathrm{C}_{16}$ have been used.

\section{Conclusion}

A laser ablation ion source has been designed, built, and tested at the LEBIT Penning trap facility at the NSCL. This source provides ions for offline studies of stable and long-lived isotopes that may be used for calibration, studies of systematic effects, and measurements of scientific interest. The LAS has enhanced the capabilities of the LEBIT facility by providing access to ions covering a wide range of mass values. Carbon cluster ions have been produced and measured with the 9.4 T Penning trap system, and additional ions produced with the LAS, such as all stable $\mathrm{Zr}$ and Mo isotopes [10], ${ }^{48} \mathrm{Ti}$ [11, and naturally abundant isotopes of $\mathrm{Cr}, \mathrm{V}$, In, Cd, Gd, and Dy have been used successfully for reference masses and for precision measurements of scientific interest, demonstrating the diverse applications available at LEBIT with the addition of this ion source.

\section{Acknowledgment}

This work was supported by Michigan State University and the National Science Foundation under Grant PHY 11-02511, and in part by the Office of Science, U.S. Dept. of Energy under Grant 03ER-41268.

\section{References}

[1] R. Ringle, S. Schwarz, G. Bollen, Int. J. Mass Spectrom. 349 (2013) 87.

[2] S. Schwarz, G. Bollen, D. Lawton, A. Neudert, R. Ringle, P. Schury, T. Sun, Nucl. Instr. and Meth. B 204 (2003) 474.

[3] P. Schury, G. Bollen, M. Block, D. Morrissey, R. Ringle, A. Prinke, J. Savory, S. Schwarz, T. Sun, Hyperfine Interact. 173 (2006) 165.

[4] M. König, G. Bollen, H. Kluge, T. Otto, J. Szerypo, Int. J. Mass Spectrom. 142 (1995) 95.

[5] K. Blaum, G. Bollen, F. Herfurth, A. Kellerbauer, H.-J. Kluge, M. Kuckein, E. Sauvan, C. Scheidenberger, Eur. Phys. J. A 15 (2002) 245.

[6] V. Elomaa, T. Eronen, U. Hager, A. Jokinen, T. Kessler, I. Moore, S. Rahaman, C. Weber, Äystö, Nucl. Instr. and Meth. B 266 (2008) 4425.

[7] C. Smorra, K. Blaum, K. Eberhardt, M. Eibach, J. Ketelaer, J. Ketter, K. Knuth, S. Nagy, J. Phys. B 42 (2009) 154028.

[8] A. Chaudhuri, M. Block, S. Eliseev, R. Ferrer, F. Herfurth, A. Martín, G. Marx, M. Mukherjee, C. Rauth, L. Schweikhard, G. Vorobjev, Eur. Phys. J. D 45 (2007) 47.

[9] A. Kellerbauer, K. Blaum, G. Bollen, F. Herfurth, H. Kluge, M. Kuckein, E. Sauvan, C. Scheidenberger, L. Schweikhard, Eur. Phys. J. D 22 (2003) 53.

[10] K. Gulyuz, J. Ariche, G. Bollen, S. Bustabad, M. Eibach, C. Izzo, S. Novario, M. Redshaw, R. Ringle, R. Sandler, S. Schwarz, A. Valverde, Phys. Rev. C 91 (2015) 055501.

[11] S. Bustabad, G. Bollen, M. Brodeur, D. Lincoln, S. Novario, M. Redshaw, R. Ringle, S. Schwarz, A. Valverde, Phys. Rev. C 88 (2013) 022501(R).

[12] D. Davies, D. Morrissey, G. Bollen, P. Lofy, S. Schwarz, J. Ottarson, Nucl. Instr. and Meth. A 569 (2006) 883.

[13] R. Singh, J. Narayan, Phys. Rev. B 41 (1990) 8843.

[14] P. Johnson, R. Christy, Phys. Rev. B 9 (1974) 5056.

[15] M. Williams, E. Arakawa, J. Appl. Phys. 43 (1972) 3460.

[16] J. Weaver, D. Lynch, C. Olson, Phys. Rev. B 10 (1974) 501.

[17] D. Dahl, Int. J. Mass Spectrom. 200 (2000) 3.

$[18]$ D. Fink, diploma thesis, Johannes Gutenberg-Universität Mainz, 2010.

[19] D. Kosimov, A. Dzhurakhalov, F. Peeters, Phys. Rev. B 78 (2008) 235433.

[20] D. Kosimov, A. Dzhurakhalov, F. Peeters, Phys. Rev. B 81 (2010) 195414.

[21] B. Bach, J. Eyler, J. Chem. Phys. 92 (1990) 358.

[22] R. Ramanathan, J. Zimmerman, J. Eyler, J. Chem. Phys. 98 (1993) 7838. 